\title{
Reflections on Public Health POLICY IN THE FOOD REGULATORY SYSTEM: CHALLENGES, AND OPPORTUNITIES FOR NUTRITION AND FOOD LAW EXPERTS TO COLLABORATE
}

\author{
MARK LAWRENCE*
}

\begin{abstract}
Diet-related diseases such as obesity, heart disease, diabetes and cancer are reaching epidemic proportions in many developed countries. Although there are increasing calls across the food regulatory system for interventions to help protect and promote public health, there is not a strong history of collaboration between public health nutritionists and food law experts in this area. This article explores the challenges facing public health nutritionists and food law experts and their opportunities to collaborate in the food regulatory system. Through a reflection on experiences with food fortification and food labelling policy debates, challenges to the objective of protecting public health and safety in the food regulatory system are identified. These challenges include: the absence of a coherent food and nutrition policy; the lack of a clear definition of what is meant by the objective 'to protect public health and safety'; capacity constraints; and limitations imposed by dominant regulatory reform agendas. Two case studies are provided to describe opportunities that are being pursued for public health nutritionists and food law experts to collaborate in the food regulatory system. The first case study describes a research project investigating reform of the Australian food regulatory system in relation to obesity prevention. The second case study describes a research proposal to review the role of nutrition in decision-making within the Australian food regulatory system. The paper concludes that, to become more effective when working across the food regulatory system to protect and promote public health, public health nutritionists and food law experts need to collaborate more strategically in research and practice.
\end{abstract}

* Associate Professor (Public Health Nutrition), World Health Organisation (WHO) Collaborating Centre for Obesity Prevention, Deakin University. 


\section{INTRODUCTION}

Food is a fundamental prerequisite for health. It has been estimated that in developed countries, diet-related diseases, including: obesity, heart disease, stroke, diabetes, and cancer, account for almost 60 per cent of preventable deaths. ${ }^{1}$ In Australia, these diseases account for approximately two thirds of the more than $\$ 85$ billion expended each year on health. ${ }^{2}$ There is a social dimension to this situation, with the burden of diet-related disease being disproportionately experienced by socially disadvantaged communities. ${ }^{3}$ From an environmental perspective, it has been estimated that the agricultural sector is responsible for 14 per cent of total greenhouse gas emissions, and, along with the food system more broadly, is a major user of water and energy resources. $^{4}$

Internationally and in many countries, there are increasing calls for food policy and food law interventions in the food regulatory system to help protect and promote public health. ${ }^{5}$ According to the Australian and New Zealand Food Regulation Ministerial Council, the 'food regulatory system' is the system of food laws and regulations across the food system. ${ }^{6}$ Historically, much attention within the food regulatory system has been focused upon developing rules and procedures to help prevent acute food safety concerns. However, far less attention has been directed towards developing rules and procedures to help prevent chronic nutrition-related health concerns. The

\footnotetext{
${ }^{1}$ World Health Organisation (WHO), 'Diet nutrition and the prevention of chronic diseases' in 'Report of a Joint WHO/FAO Expert Consultation', Technical Report Series 916 (2003), WHO, Geneva.

${ }^{2}$ Australian Institute of Health and Welfare, Australia's Health 2008 (2003), AGPS, Canberra.

${ }^{3}$ World Health Organisation (WHO), Commission on the Social Determinants of Health, Closing the gap in a generation: Health equity through action on the social determinants of health (2008), WHO, Geneva.

${ }^{4}$ Anthony Costello et al, 'Managing the health effects of climate change' (2009) 373 The Lancet 1693.

${ }^{5}$ World Health Organisation (WHO), Global strategy on diet, physical activity and health (2004) World Health Organisation ('Global Strategy') <http://www.who.int/ dietphysicalactivity/strategy/eb11344/strategy_englishweb.pdf> at 31 January 2009;

Commonwealth of Australia, National Preventative Health Taskforce, Technical Report No 1: Obesity in Australia: a need for urgent action (2008) ('Obesity in Australia') $<$ http://www.preventativehealth.org.au/internet/preventativehealth/publishing.nsf/Content/E2 33F8695823F16CCA2574DD00818E64/\$File/obesity-jul09.pdf> at 22 October 2009; Marion Nestle, 'What President Obama can do in the USA' (2009) 12(3) Public Health Nutrition 433.

${ }^{6}$ Australian and New Zealand Food Regulation Ministerial Council, Overarching Strategic Statement for the Food Regulatory System (2008), Department of Health and Ageing $<$ http://www.health.gov.au/internet/main/publishing.nsf/Content/FB280282EE51D887CA256 F190003B038/\$File/Overarching\%20Strategic\%20Statement.pdf $>$ at 20 May 2009.
} 
paradox is that, relative to food safety concerns, chronic nutrition-related health concerns arise far more frequently and impose a more substantial economic burden across society. ${ }^{7}$

In spite of the increasing recognition of the need for food regulatory approaches to help protect and promote public health, there is a lack of evidence that this is being translated into effective practice by public health nutritionists. The argument of this paper is that, for public health nutritionists, maintaining the status quo in relation to how they develop and implement food policy interventions across the food regulatory system is not an option if they aim to successfully tackle the epidemic of diet-related chronic diseases and address related social and environmental considerations. Instead, they need to collaborate more strategically with food law experts to exploit food policy opportunities. In making this argument, this paper: provides a background description of the disconnect between public health nutritionists and food law experts; briefly reflects on core public health policy debates in the Australian food regulatory system; identifies several core challenges to protecting public health in the food regulatory system that emerge from the reflection; and then describes two case studies of collaborative ventures between public health nutritionists and food law experts where the opportunity is being taken to work together across the food regulatory system to protect public health.

\section{Background - The Disconnect Between Public HEALTH NUTRITIONISTS AND FOOD LAW EXPERTS}

For many decades public health nutritionists have been actively involved in food policy activities across the food regulatory system with the aim of protecting and promoting public health. However, such public health nutritionists have tended to rely predominantly on relatively 'soft' policy instruments, for example education interventions, to achieve policy objectives. When they have pursued the development of regulatory interventions ('hard' policy instruments) they have too often done so by attempting to 'go it alone', that is, without sufficient input from food law experts. With increasing epidemics of diet-related disease and emerging social and environmental challenges, such practices have evidently not been effective in helping to make 'healthier choices the easier choices' - the theme of many food and nutrition policies. A key explanation of their failure is that education interventions primarily benefit those from higher socio-economic backgrounds and have limited impact on non-behavioural factors, through

\footnotetext{
${ }^{7}$ Mike Rayner and Peter Scarborough, 'The burden of food related ill health in the UK' (2005) 59 Journal of Epidemiology and Community Health 1054.
} 
promoting the availability of, and accessibility to, a healthy food supply, which is important for all people in society. For instance, the National Preventative Health Taskforce stresses that the obesity epidemic is less the result of mass gluttony and more an example of 'market failure', and therefore recommends the need for sophisticated regulatory interventions in the marketplace to help correct this failure. ${ }^{8}$ The Taskforce's argument is that, whereas the market may be a commercial success delivering benefits for some, overall it has failed society because much of its commercial success has been derived from promoting over-consumption, particularly of poor nutritional quality foods. This has had a negative impact on the population's health profile. A secondary explanation is that public health nutritionists generally lack the competencies needed to prepare and implement regulatory proposals. For example, they lack knowledge regarding where and when to intervene in the regulatory environment and how to draft legislative amendments.

Conversely, potential problems can arise when non-public health nutritionists, who may misunderstand nutrition principles and concepts, attempt to use food regulatory approaches to influence food choices in order to help protect and promote public health. For instance, the relationship between poor food choices and diet-related disease is often thought of as the nutrition equivalent of the relationship between cigarette smoking and lung cancer and/or emphysema. As a consequence, it is often assumed that regulatory approaches to tobacco control might readily transfer into the public health nutrition sphere. Certainly, there are some relevant comparisons to be made between the tobacco and food industries, such as the common characteristics of many of the marketing strategies of product manufacturers in both areas, and their capacity to exert influence over political debates. However, there are fundamental differences that, if not considered, may result in regulatory approaches being counterproductive. For example, while there is compelling evidence of a direct cause and effect relationship between cigarette smoking and poor health, there is a lack of evidence to demonstrate that individual foods are directly associated with chronic diet-related disease causation. Therefore, attempts to introduce regulatory measures specific to individual foods can be fraught with danger. For example, the introduction of a so-called 'fat tax' - the imposition of a tax on high fat-containing foods - could readily lead to a number of anomalies. For instance, a potential unintended consequence of a fat tax is that regulators may tax foods such as nuts, which, although approximately 50 per cent of their energy content is derived from fat, are recommended as core components of a healthy diet.

\footnotetext{
${ }^{8}$ See National Preventative Health Taskforce, Obesity in Australia, above n 6.
} 


\section{Reflections on Core Public Health Policy DEBATES IN THE FoOd REgULATORY SySTEM}

Food fortification and food labelling are core public health policy issues in the food regulatory system. Food fortification has a direct bearing on the composition of affected food products and subsequently on the dietary exposure to the fortificant of those individuals who consume the food product. Food labelling provides a communication tool that, among other functions, may inform citizens about the nutrition, health or related properties of a food. The following section provides brief descriptions of debates associated with particularly vexatious aspects of these complex policy issues. The descriptions illustrate how public health was interpreted and applied, and how nutrition science was taken into account in decision-making.

When the then Australian National Food Authority (NFA), the forerunner to Food Standard Australia New Zealand (FSANZ), was established in August 1991, it inherited a number of food standards matters that were carried over from the previous National Health and Medical Research Council (NHMRC) food standards setting system. Among the inherited matters was food Standard A9 - Vitamins and minerals, the food standard that regulates the addition of essential nutrients to food products. During the early 1990s in Australia, an increasing number of fortified food products, such as fortified breakfast cereals, were being introduced into the marketplace for no apparent health reason, and with different combinations and levels of added nutrients. In 1992, the NFA commenced a review of Standard $A 9^{9}$ to clarify which products could be fortified, with which nutrients they could be fortified, and what levels of fortification were required. The review adopted a public health policy principle requiring the existence of scientific evidence to demonstrate a public health need, prior to permitting the fortification of food products.

The Food Authority's adoption of this public health policy principle of 'demonstrated need' as the basis for its review of Standard A9 led to a particularly heated and truncated debate about what was meant by the protection of public health and safety, and what should constitute the role of the Authority in developing and implementing policy in this area. The majority of public health agencies and practitioners supported the Food Authority's policy principle, arguing that there was no evidence of a public health need for many of the fortified products in the marketplace up to that time, and that fortification was instead being abused to promote marketing of

\footnotetext{
${ }^{9}$ National Food Authority (NFA), 'Full Assessment Report, Proposal 24 - Vitamins and Minerals, National Food Authority' (2002), NFA, Canberra.
} 
so-called 'junk' foods. ${ }^{10}$ In contrast, a number of food manufacturers took exception to the NFA's policy approach to the review of Standard A9, arguing that the requirement for an evidence-based justification of health needs prior to fortification would restrict trade opportunities as well as stifle food product innovation. ${ }^{11}$ During this period certain food manufacturers were alleged to be particularly aggressive in lobbying senior government officials to oppose the Food Authority's review and challenge the work of the NFA staff. For instance, according to a government Member of Parliament directly involved with the NFA at the time:

There was a danger that [food company X] would leave Australia ... you don't allow yourself to be blackmailed by international companies, but on the other hand why would you want $[\mathrm{X}]$ to get out of Australia merely because they want to put vitamins in [their products]. If it's dangerous to health, that's different. ${ }^{12}$

In addition to illustrating the degree and nature of lobbying being undertaken by one food manufacturer, the Member of Parliament's comment is revealing in terms of how decision-makers within the food regulatory system were interpreting the objective of protecting public health and safety. Specifically, public health and safety risk was being interpreted in terms of acute safety concerns. In 1994, the then Australian Food Standards Council voted to reject its own Food Authority's policy recommendations related to the addition of nutrients into food products. Instead, the Council resolved that, unless there was evidence of harm, trade opportunities and innovation should not be restricted. This decision then placed the burden of proof on public health interests to demonstrate harm, rather than on food manufacturers to demonstrate a public health need. ${ }^{13}$ Through a process known as 'grandfathering', the Council also decided to provide special permissions for those food products (notably breakfast cereals) that had previously been fortified to continue this practice irrespective of their fat, salt and/or added sugar content. This decision was seen by many public health nutritionists as further ignoring public health recommendations such as the dietary guidelines for Australians.

In a separate, more recent, public health policy debate within the food regulatory system, FSANZ initiated 'Proposal P293 - Nutrition, Health and

\footnotetext{
${ }^{10}$ National Food Authority (NFA), 'NFA 16 Preliminary Inquiry Report, Proposal 24 Vitamins and Minerals, National Food Authority’ (1993), NFA, Canberra.

${ }^{11}$ Ibid.

${ }^{12}$ Key informant 19 as cited in Mark A Lawrence, Folate Fortification: A Case Study of Public Health Policy-Making (PhD thesis, Deakin University, 2002) 245.

${ }^{13}$ Key informant 2 in Lawrence, ibid.
} 
Related Claims' to bring the diversity of food labelling issues encompassed by such claims under one food standard. ${ }^{14}$ Among the many types of claim are: nutrition content claims, such as 'source of calcium'; general level health claims, such as 'food X is a good source of calcium and calcium helps build strong bones and teeth'; and high level health claims, such as 'food X is a good source of calcium and will help prevent osteoporosis'. In attempting to bring the issues raised by the claims under one standard, the Proposal has had to contend with a range of questions: Should health claims be permitted on food products? If so, what level of scientific evidence is required to substantiate such a claim? And, are there certain food products for which nutrition content claims and/or health claims should not be permitted?

Proposal P293 covers nutrition, health and related claims information about which there are strongly held differences of opinion among stakeholders. Most public health agencies and practitioners have argued the need for socalled 'disqualifying criteria' to determine those food products not eligible to access nutrition content claims. For instance, they argue that a high fatcontaining product should not be allowed to be advertised as a good source of any particular nutrient - and, by implication, as a healthy food - since such a claim is inconsistent with the dietary guideline message to "moderate fat intake'. ${ }^{15}$ Conversely, many food manufacturers argue that there should be no restrictions on the use of nutrition content claims on food products, irrespective of the consistency of that food product with the dietary guidelines. ${ }^{16}$ They state that such claims are simply technical statements relaying factual food composition information to citizens. In addition, they contend that all nutrition content claims are a legitimate nutrition education tool, and represent a practical way for food manufacturers to inform citizens about healthy eating.

In its 'Preliminary Final Assessment Report' for 'Proposal P293 - Nutrition, Health and Related Claims', ${ }^{17}$ FSANZ outlines that there will be disqualifying criteria for general and high level health claims, but not for nutrition content claims. As a consequence of these policy decisions, many food products are permitted to be fortified and to display nutrition content claims, irrespective of

14 Food Standards Australia New Zealand at <http://www.foodstandards.gov.au/ standardsdevelopment/proposals/proposalp293nutritionhealthandrelatedclaims/index.cfm $>$ at 22 October 2009.

15 National Health and Medical Research Council (NHMRC), Dietary Guidelines for Australian Adults (2003), AGPS, Canberra.

${ }^{16}$ See Dick Wells, Talk to IBC Conference, 29 April 2003, Australian Food and Grocery Council <http://www.afgc.org.au/cmsdocuments/IBC\%20Conference.pdf $>$ at 21 May 2009.

17 Food Standards Australia New Zealand <http://www.foodstandards.gov.au/standards development/proposals/proposalp293nutritionhealthandrelatedclaims/p293preliminaryfinal35 02.cfm> at 22 October 2009. 
their consistency with dietary guideline criteria. A situation now exists where products such as Kellogg's Coco Pops are advertised as being a healthy food choice for children on the basis of their nutrient composition (predominantly derived from fortification processes), despite being highly processed, expensive and consisting of over one-third (36 per cent by weight) sugar. Although the reviews of Standard A9 and Proposal P293 were undertaken independently and at different time periods, they are nonetheless intrinsically related in their application and in their implications for the food marketplace and ultimately public health. For instance, the ability to make nutrition, health and related claims is frequently contingent on the prior addition of vitamins and minerals to food products - as the Coco Pops marketing strategy indicates. Some stakeholders argue that the public health policy decisionmaking processes within the food regulatory system are not sufficient to account for broad trends in the food supply and subsequent impacts on public health. For example, the Chairperson of the NFA at the time of the review of Standard A9 believed that it was inevitable that food fortification and food labelling developments were intertwined and that decision-makers needed to pay more attention to the broader public health implications beyond immediate safety concerns. She commented that the review of Standard A9

was a 'stalking horse' for the next big fight which was going to be full health claims. So it was a combination of medicalising the food if you will, getting the stuff into the food, so that the later claims could be made about the health benefits of that food. ${ }^{18}$

\section{Challenges in the Food Regulatory System for Protecting and Promoting Public Health}

The reflections on the reviews of Standard A9 and Proposal P293 highlight a number of challenges faced by those intervening in the food regulatory system in order to protect and promote public health. The challenges that are discussed in this section are: the absence of a coherent food and nutrition policy; the lack of a clear definition of what is meant by the objective 'to protect public health and safety'; capacity constraints; and limitations imposed by dominant regulatory reform agendas.

\footnotetext{
${ }^{18}$ Ibid, key informant 2, above n 15, 234.
} 


\section{A The Absence of a Coherent Food and Nutrition Policy}

In 1992 the Commonwealth government launched its national food and nutrition policy. ${ }^{19}$ The centrepiece of the policy was the 'Dietary Guidelines for Australians'. The policy received initial funding to develop and implement several pilot public health nutrition projects. However, within five years there was no budget allocated to the policy and, by the end of the 1990s, it was effectively non-existent and has not been replaced. The absence of a coherent and overarching food and nutrition policy is a key reason for the disconnect between dietary guideline recommendations promulgated from within the health sector, and the food law and policy decisions associated with the food regulatory system. For example, the Food Authority is hampered in its ability to situate individual food standards decisions within a broader public health context.

\section{B The Lack of a Clear Definition of the Objective: 'to protect public health and safety'}

These reflections indicate that, despite the protection of public health and safety being the primary objective in the setting of food standards, there is no clear definition of what this objective means in policy practice. Different stakeholders have different views on how it should be interpreted and applied. For example, what is the difference between protecting and promoting public health, and between protecting public health and protecting safety in the food regulatory system? In relation to food fortification and food labelling policy decisions, it is apparent that the objective was interpreted in terms of protecting against relatively short term and acute risks to public safety. The decisions were informed by evidence from toxicological and microbiological studies and generally did not address more chronic public health concerns informed by evidence from nutrition studies. In isolation, individual policy and food standards decisions may appear to have a relatively benign impact on public health. However, when examining the effects of multiple policy decisions, inconsistencies with public health recommendations can emerge. The decision-making processes of the food regulatory system are not protecting citizens from the proliferation of heavily marketed and highly processed, expensive foods with high fat, sugar and salt content.

\footnotetext{
${ }^{19}$ Commonwealth Department of Health and Human Services, National Food and Nutrition Policy, Commonwealth of Australia, AGPS, 1992.
} 


\section{Capacity Constraints}

The food regulatory system is a highly politicised setting. This is an especially relevant consideration in a country such as Australia which is a net food exporter and has a vested interest in developing and marketing value-added highly processed food products. The reflections indicate limitations in the capacity of current policy processes to account for public health and safety within the food regulatory system. They also illustrate that public health interests are often strongly contested by food manufacturers and trade interests. It would be naïve to assume that decision-makers will automatically defer to public health interests in resolving policy debates. For instance, by adopting a policy position in relation to Standard A9 requiring evidence of harm rather than evidence of efficacy, the National Food Standards Council has shifted the burden of proof onto the public health community which must now positively prove its case, and away from food manufacturers.

\section{Limitations Imposed by Dominant Regulatory Reform Agendas}

The evolution of the roles, responsibilities and structures of the food regulatory system has occurred against a series of regulatory reforms and governmental agreements. The more prominent reviews and agreement have been:

- 1995, 1997, 2004: Council of Australian Governments, Principles and Guidelines for National Standard Setting and Regulatory Action; ${ }^{20}$

- 1998: the Blair Review - Food: A Growth Industry. Report of the Food Regulatory Review; ${ }^{21}$

- 2002: Council of Australian Governments - Intergovernmental Agreement; ${ }^{22}$

- 2006: The Banks Review - Rethinking Regulation, Report of the Taskforce on reducing regulatory burdens on business, ${ }^{23}$ and

\footnotetext{
${ }^{20}$ Council of Australian Governments, 'Principles and Guidelines for National Standard Setting and Regulatory Action', 1995, 1997, Canberra. Amended again in 2004.

${ }^{21}$ Food Regulation Review Committee, Food: a Growth Industry. Report of the Food Regulation Review ('The Blair Review') (1998), AGPS, Canberra.

22 Council of Australian Governments, 'Inter-Governmental Agreement' (2002) $<$ http://www.foodstandards.gov.au/_srcfiles/31_Fina_signed-FRA2002.pdf $>$ at 11 May 2009

${ }^{23}$ Food Regulation Review Committee, Rethinking Regulation: Report of the Taskforce on Reducing Regulatory Burdens on Business ('The Banks Review') (2006), AGPS, Canberra.
} 
- 2008: Victorian Competition and Efficiency Commission review Simplifying the Menu: Food regulation in Victoria. ${ }^{24}$

As indicated by their titles, the reforms and agreements have been framed within a neoliberal agenda of streamlining and reducing regulation (or socalled 'red tape'). These reforms have dictated the context within which internal food regulatory system decision-making has been, and continues to be, undertaken. A common theme in all the reviews has been the promotion of opportunities for food manufacturers to innovate and add value to their products, especially with a view to increasing export markets. Invariably this agenda has created uncertainty about what is meant by protecting public health and safety in the work of the food regulatory system and has resulted in challenges to the balancing of economic and public health interests in food policy and food law.

\section{EXAmples Where OpPortunities To Collaborate ARE BEING UNDERTAKEN}

In this section two case studies are provided to illustrate food policy and food law research activities that are combining the expertise of public health nutritionists and lawyers in helping to promote and protect public health in the food regulatory system. The first case study represents a currently funded research project to investigate reform of the food regulatory system to support obesity prevention. The second case study represents a research proposal to provide evidence to help review the role of nutrition in the decision-making processes of the Australia food regulatory system.

\section{A Case study 1: A Comprehensive Regulatory Strategy for Obesity Prevention in Australia}

In 2007 an interdisciplinary team consisting of law and public health nutrition researchers from the Department of Epidemiology and Preventive Medicine at Monash University and the WHO Collaborating Centre for Obesity Prevention at Deakin University respectively, were awarded an NHMRC Strategic Award (Preventative Healthcare and Strengthening Australia's Social and Economic Fabric) to investigate reform of the food regulatory system. The current writer is a member of this team. The specific aim of this research is to build the evidence base for developing regulatory approaches to

\footnotetext{
${ }^{24}$ State of Victoria, Simplifying the menu: Food Regulation in Victoria: Victorian Competition and Efficiency Commission's Final Report: Victorian Government Response (2008).
} 
help prevent obesity and to reduce socio-economic inequalities in obesity prevalence.

As a first step in the research process, the public health nutritionists have constructed so-called 'analysis grids' to help strategically organise the sector or setting in which particular policies apply and the level of government that is responsible for administering that policy. ${ }^{25}$ The grids provide a comprehensive approach to mapping the policy environment related to obesity, and a tool for identifying policy gaps, barriers and opportunities. Specifically, the grids are being applied to identify 'policy areas' across the food supply system and environments related to physical activity. ${ }^{26}$ Systematic reviews of the health and policy literature have been conducted to identify the evidence base for the specific policy options within each of the major policy areas. These policy options are being refined via focus groups and one-on-one interviews with government representatives, academics and other key stakeholders to establish the most 'promising policy interventions'. Potential policy interventions have been developed and assessed across a range of policy areas including transport, the built environment, agriculture, food distribution, food marketing and food retail.

The project's legal experts are assessing the regulatory and legal pathways for the development and implementation of each promising policy intervention. Laws related to these policy interventions will be comprehensively analysed and prioritised, and options proposed for legislative reform. Much time will be devoted to working closely with those within the bureaucracy who administer the law and legislators who together make the relevant policy decisions so that they may gain an understanding of the rationale behind each recommendation. The evidence base and the implementation pathways are being developed contemporaneously and will be widely promoted to governments. In order to achieve implementation of any proposed model law, such law will need to be practical and beneficial, and seen to be so.

The immediate outcome of this research project will be a model regulatory framework for obesity prevention. The long term outcomes, if the recommendations are implemented, will be changes in the food supply, and dietary and physical activity patterns which will contribute to reduction in obesity and the reduction in the socio-economic gradient in obesity prevalence.

\footnotetext{
${ }^{25}$ G Sacks, B Swinburn and M Lawrence, 'Obesity Policy Action framework and analysis grids for a comprehensive policy approach to reducing obesity' (2008) 10(1) Obesity Reviews 76.

${ }^{26} \mathrm{G}$ Sacks, B Swinburn and M Lawrence, 'A systematic policy approach to changing the food and physical activity environments to prevent obesity' (2008) 5 Australia and New Zealand Health Policy 13.
} 


\section{B Case Study 2: Integrating Nutrition into Food Law and Policy - An Interdisciplinary Analysis}

In 2008-2009 an interdisciplinary team consisting of researchers from the WHO Collaborating Centre for Obesity Prevention, the Deakin University Law School, the Deakin University Business School and the National Centre for Epidemiology and Public Health at the Australian National University was formed. Again, the current writer was a member of this team. Its purpose was the collaborative preparation of an Australian Research Council Discovery grant proposal with the goal of undertaking interdisciplinary analysis to integrate nutrition into food law and policy. It proposes to combine methods of traditional legal analysis with social considerations and economic analysis in the area of public health in order to provide a nationally harmonised framework for integrating nutrition considerations into the decision-making process when policy and food standards are being set.

Currently, governments at state, national and international level are struggling with the dual challenges of defining: (a) the nature and scope of public health considerations in regulating the food industry, and (b) the role of food regulations in modifying food production and marketing to support public health nutrition policy objectives. The research proposes to conceptualise and articulate these challenges, so as to provide research-driven, novel and innovative options and approaches for decision-makers to consider in their policy-making work within the food regulatory system. The research will provide options and approaches that can be applied to legal and policy practice by:

i) Providing guidelines to help promote public health nutrition

There are now many calls for new ways of conceptualising the basis for legislation to help mitigate and prevent nutrition-related diseases and thereby promote public health. ${ }^{27}$ For example, the Australia and New Zealand Food Regulation Ministerial Council ${ }^{28}$ has commissioned an 'independent, comprehensive review of food

\footnotetext{
${ }^{27}$ Lawrence O Gostin, 'Law as a Tool to Facilitate Healthier Lifestyles and Prevent Obesity' (2007) 297(1) Journal of the American Medical Association 87.

Roger Magnusson, 'What's Law Got to Do with It? Part 2: Legal Strategies for Healthier Nutrition and Obesity Prevention' (2008) 5 Australia and New Zealand Health Policy 11.

${ }^{28}$ Australia and New Zealand Food Regulation Ministerial Council Joint Communiqué, Food Ministers Consider Food Labelling Review 24 October $2008<\mathrm{http} / / \mathrm{www}$. health.gov.au/internet/main/publishing.nsf/Content/AF645609D3B3E340CA2574F2000122C 4/\$File/Communique\%2024\%20october.pdf> at 9 February 2009.
} 
labelling law and policy ${ }^{29}$ so that such labelling is more responsive to public health nutrition considerations. The research will:

(a) critically review food composition and food labelling standards for their capacity to promote the development of healthy food products and the provision of adequate food information, and;

(b) develop regulatory impact statements ${ }^{30}$ which, in addition to outlining the impact on established interests (for example, trade and economic interests), will predict the effect on public health nutrition of different regulatory options.

ii) Reviewing risk analysis protocols to help protect public health nutrition

The Australian food marketplace is undergoing rapid expansion, with a plethora of new products being introduced each year. Many are fortified with novel amounts of nutrients and/or ingredients; some are genetically modified; others involve nanotechnology. Frequently these products are marketed as having particular nutritional benefits, which help reduce risk of disease or enhance health. A clearly articulated definition of the protection of public health and safety, and of the criteria for their protection are required to assess potential risks and benefits to public health nutrition associated with the consumption of these products. Absence of an appropriate definition of public health and safety has meant that regulators have concentrated only on immediate food safety concerns (food hygiene, microbiology, toxicology). Rather than focusing on food safety in its strict sense, the research will (through the use of risk analysis protocols) review the scope of existing legislative provisions aimed at protecting public health. It will do so in accordance with criteria reflecting the most pressing contemporary public health nutrition problems, namely diet-related chronic diseases.

\footnotetext{
${ }^{29}$ Australia and New Zealand Food Regulation Ministerial Council, Joint Communiqué, 24 October $2008<$ http://www.health.gov.au/internet/main/publishing.nsf/Content/6C8C69E 90DF4BFF0CA2574EC00191687/\$File/dept241008.pdf $>$ at 22 October 2009.

${ }^{30}$ Australia and New Zealand Food Regulation Ministerial Council, Principles and Protocols for the Development of Food Regulation Policy Guidelines 2 May 2008 $<$ http://www.health.gov.au/internet/main/publishing.nsf/Content/2087CDEAEE7C703CCA25 6F190003AF4B/\$File/Principles\%20and\%20Protocols\%20for\%20the\%20Development $\% 20 \mathrm{o}$ f\%20Food\%20Regulation\%20Policy\%20Guidelines\%20-\%202\%20May\%202008.pdf >;

Council of Australian Governments (COAG), Intergovernmental Food Regulation Agreement 3 July $2008<$ http://www.coag.gov.au/intergov_agreements/index.cfm> at 10 February 2009.
} 
The conceptual framework underpinning this proposal positions the food regulation-public health nutrition relationship as existing in two orientations. First, food regulatory measures can serve as a policy instrument available to help promote public health nutrition objectives, particularly when markets fail. Second, when applying the objective, 'to protect public health and safety' in the development of policy and food standards, public health nutrition criteria can be integrated into the decision-making process for risk analysis protocols.

The researchers will ask: what are the legal, conceptual, economic, and social equity barriers to the legal integration of nutrition into the food regulatory system in Australia? To answer this research question, the project will combine economic, legal, public health, and sociological research methods. The research design and methods will follow the following sequence of integrated steps to achieve the aims of the proposal:

(i) The development of a nutrition-oriented definition of public health as a tool to critically analyse the food regulatory system;

(ii) The examination of international regulatory models;

(iii) The conduct of key informant interviews to ascertain public health regulation best practice; and

(iv) The building of economic models to identify benefits and compliance costs associated with recommended changes in the law.

Through critical examination of the existing system, the research will be able to:

(i) pinpoint discrepancies and gaps in each Australian jurisdiction;

(ii) provide comparative tables of existing legislative provisions in Australian jurisdictions; and

(iii) propose guidelines for adapting and re-modelling the existing legislative provisions that were initially enacted in an ad hoc manner to regulate food trade and food safety in order to incorporate public health nutrition considerations.

It will also suggest ways of harmonising the relevant legislation. This conceptual, evidence-based approach will make a substantial contribution to advancing the food law and policy knowledge-base, especially in its application to the promotion and protection of public nutrition. It will also help to ensure that the Australian food regulatory system follows international best practice in its food law and policy. 
The proposed research will result in the following scientific, economic and social benefits for the Australian population:

(i) An evidence-based framework (with public health and social dimensions and including risk analysis protocols and regulatory impact statements) to inform decision-making and policy practice within the future food regulatory system;

(ii) Anticipated cost savings from the reduction of the illness burden on the Australian health system; and

(iii) A projected reduction in the inequitable distribution of the burden of diet-related chronic disease across the population.

These case studies provide powerful examples of experts in public health nutrition, law, economics and epidemiology combining to seek to promote and protect public health within the food regulatory system. Such an interdisciplinary research project supports the call by policy-makers internationally $^{31}$ and nationally ${ }^{32}$ for legislative frameworks and regulatory provisions to protect and promote public health nutrition objectives.

\section{CONCLUSION}

The food regulatory system is a powerful setting for protecting and promoting public health nutrition. In the future the system will continue to respond to a variety of (often competing) public health, economic, social, technological and political interests when setting policy and food standards. With an unprecedented expansion of global and national food systems and a proliferation in new food products, the role of the food regulatory system to protect and promote public health nutrition will become even more critical. In particular, there is a need for an unambiguous definition of the objective 'to protect public health and safety' - a definition that integrates evidence from nutrition science into the decision-making processes of the food regulatory system.

The history of public health nutritionists working in the food regulatory system has been one of mixed success. Whereas they have the technical expertise to inform food policy and regulations, and have skills in identifying problems and recognising potential solutions, they are less competent at knowing how to act within the context of food law and legislative frameworks. The challenges and opportunities identified in this paper highlight the fact that, in order to become more effective when working across

\footnotetext{
${ }^{31}$ See WHO, Global Strategy, above n 5.

${ }^{32}$ See National Preventative Health Taskforce, Obesity in Australia, above n 6.
} 
the food regulatory system and hence to protect and promote public health, public health nutritionists and food law experts need to collaborate more strategically in research and practice. 